\title{
ANÁLISIS ESTADISTICO DE DAT'OS DE ENCUESTAS. TRATAMIENTO CONEXO DE RESPUESTAS A PREGUNTAS ABIERTAS Y CERRADAS
}

\author{
Mónica Bécue Bertaut \\ Departament d'Estadistica i Investigació Operativa \\ Facultat d'Informàtica de Barcelona (UPC)
}

Resumen

El sociólogo se encuentra muchas veces enfrentado, en la recogida de datos con información textual, sea con las preguntas abiertas de una gran encuesta, sea con entrevistas, sea con otros textos de fuentes de datos secundarios. Tanto con fines exploratorios o de clasificación previa, como a la bora de la comprobacion de determinadas hipótesis el método, y el programa informático SPAD.T correspondiente, constituye un aitil importante para el tratamiento de textos. En particular permite confrontar los resultados obtenidos del análisis estadistico de textos con otras variables estructurales provenientes de las grandes encuestas interviniendo como variables ihustrativas.

Restum

El socioleg es troba moltes vegades enfrontat, a la recollida de les dades amb informació textual, be sigui amb les preguntes obertes d'una gran enquesta, bé sigui amb entrevistes, bé sigui amb altres textos de fonts de dades secundàries. Tant amb finalitat exploratories o de classificacio prèvia com a lhora de la comprovació de determinades hipotesis, el mètode i el programa informàtic SPAD.T corresponent, constitueixen una eina important pel tractament de textos. Especialment, permet confrontar els resultats obtinguts de l'anàlisi estadistica de textos amb altres variables estructurals provinents de les grans enquestes intervenint com a variables il.hustratives.

Abstract

The sociologist can be confronted with textual information, during data gathering, in large surveys, in interviews or in other secondary sources. The SPAD. $T$ is a useful and important tool in dealing with texts, in exploratory aims or in a priori classifications, as well as the verifying moment of the bypothesis of the method. In particular, it allows to confront results obtained from text statistical analysis and other structural variables coming from large surveys, being introduced as ilustrative variables. 
"Papers": Revista de Sociologia

Los métodos estadísticos multidimensionales de análisis de grandes tablas de datos permiten tratar las respuestas a preguntas cerradas y abiertas de forma simultánea.

Entre estos métodos, los de análisis de correspondencias, correspondencias simples y múltiples, cumpien un papel privilegiado. Métodos de descripción de las tablas de contingencia (o tablas cruzadas) y de ciertas tablas binarias (matriz de respuestas a cuestionarios, por ejemplo), proporcionan una representación gráfica de las asociaciones entre las líneas y las columnas.

Son métodos muy difundidos desde los trabajos de J. P Benzecri (Benzécri 73), J.P. Fénelon, L. Lebart, A. Morineau (Lebart, Morineau, Fénelon 84), Y. Escoufier (Escoufier 85), J.M. Bouroche, G. Saporta (Bouroche, Saporca 80 ), de Volle (Volle 80) que los han estudiado de forma sistemática como herramientas flexibles para el tratamiento de datos multidimensionales.

Los métodos de clasificación automática pueden complementar la descripción gráfica obtenida. La reagrupación de los individuos en clases homogéneas efectuadas permite simplificar y sintetizar las representaciones gráficas.

Más recientemente, J.P Benzecri (Benzecri 81), L. Lebart y A. Salem (Lebart, Salem 88) han introducido la aplicación de estos métodos en el terreno textual. Esta aplicación ofrece una nueva aproximación a los datos textuales. Es una aproximación esencialmente diferencial que describe los contrastes entre los textos o las respuestas de encuesta (respuestas individuales o grupos de respuestas) y que confronta las respuestas abiertas y cerradas.

El sistema informático SPAD.T (Lebart, Morineau, Bécue 89) automatiza la aplicación de estos métodos, integrándolos en un mismo entorno y constituye, así, una herramienta para el tratamiento comparativo de textos. Permite trabajar a partir de los datos brutos, sin precodificación ni manipulación previas y analizar conjuntamente respuestas abiertas y cerradas.

\section{APORTACION ESPECIFICA DE LAS PREGUNTAS ABIERTAS}

Las respuestas abiertas son, todavía, poco utilizadas. El análisis de la información que dichas respuestas proporcionan es a la vez complejo y costoso. No obstante, en ciertos casos es imposible sustituir una pregunta abierta por una pregunta cerrada ya que estos dos tipos de preguntas aportan informaciones de naturaleza muy distinta y, por lo tanto, difícilmente comparables.

Por esta y otras razones, el uso de preguntas abiertas puede ser indispensable. Facilita la exploración de dominios mal conocidos, para los cuales todas las posibles respuestas cerradas no son conocidas a priori. Reduce el tiempo de la entrevista, cuando una sola pregunta abierta sustituye a varias listas de ítems. Permite explicitar las respuestas a preguntas cerradas (con la cues- 
tión complementaria ¿Por qué?) y, así, saber si los entrevistados han entendido los valores de la pregunta cerrada de la misma forma.

\section{EJEMPLO DE APLICACION}

El ejemplo utilizado en este artículo proviene de una encuesta efectuada sobre 2000 individuos representativos de los franceses mayores de edad residentes en Francia continental (Lebart , Houzel 80). El cuestionario intenta cubrir varios dominios de estudio tales como la familia, las condiciones de vida y las aspiraciones de los franceses. Comporta 410 preguntas cerradas y 6 preguntas abiertas.

La pregunta abierta escogida en este ejemplo tiene el libelado siguiente: "Le nombre de divorces augmente actuellement en France; à quoi est due, selon vous, cette augmentation?».

Las respuestas abiertas de los 2.000 individuos se graban sobre un soporte magnético sin otra codificación previa que la marca de fin de respuesta.

\section{UNIDADES DE TRATAMIENTO ESTADÍSTICO}

El sistema SPAD.T considera dos unidades para el tratamiento estadístico del corpus: la forma gráfica y el segmento repetido.

La forma gráfica será la unidad de base. Viene definida como sucesión de caracteres no delimitadores (en general letras) comprendidos entre dos delimitadores (blancos y signos de puntuación). A una misma forma léxica (unidad de lengua definida en el diccionario) pueden corresponder varias formas gráficas - femenirto y masculino de un mismo adjetivo, flexiones distintas de un verbo. Inversamente, una misma forma gráfica puede referirse a formas léxicas distintas.

La conservación del genero de un adjetivo, del tiempo de un verbo no sólo facilita la completa automatización del tratamiento sino que, sobre todo, mantiene una información no despreciable, la información contenida en la propia utilización del género, de un tiempo pasado en lugar del presente, etc.

La no identificación de homógrafos (como del verbo comer y como conjunción, por ejemplo) no es, en general, un inconveniente grave porque las palabras no están siendo tratadas de forma aislada. No obstante, el tusuario puede utilizar la edición de concordancias--edición de todos los contextos de todas las ocurrencias de una forma - para distinguirlos y introducir esta información en la cadena de tratamiento.

La segunda unidad estadística considerada es el segmento de frase repetido. 
"Papers": Revista de Sociologia

Es una unidad de recuento compuesta por varias formas contiguas. Esta nueva unidad estadística, introducida por A. Salem (Salem 82, Salem 87), permite tomar en cuenta el contexto de las formas.

\section{GLOSARIO DE FORMAS Y SEGMENTOS REPETIDOS}

El tratamiento preliminar del corpus constituido por las 2000 respuestas a la pregunta abierta sobre el divorcio consiste en identificar estas unidades, contarlas y construir las tablas léxicas o segmentales que serán sometidas al análisis de correspondencias.

Se obtiene así una primera información sobre el corpus: su longitud y el número de formas distintas que lo componen, respectivamente $18.620 \mathrm{ocl}$ rrencias y 1.667 formas distintas en el ejemplo.

La tabla 1 representa las 159 formas empleadas al menos 15 veces en todo el corpus. La forma más frecuente es de, empleada 1.180 veces. La primera forma plena es vie, pronunciada 341 veces. Se puede notar que las formas femme y femmes, pronunciadas respectivamente 220 y 205 veces, aparecen como las tercera y cuarta formas significativas mientras que las formas homme y hommes se encuentran empleadas solamente 32 y 18 veces respectivamente.

En la tabla 2, se muestran los segmentos repetidos del corpus. Están seleccionados en función de umbrales de frecuencia distintos según la longitud del segmento y listados en orden lexicográfico.

\section{TABLA 1}

Glosario de las formas más frecuentes

DICTIONNAIRE DES MOTS

\begin{tabular}{|c|c|c|c|c|c|c|c|}
\hline NLIM, & MOTS EMPLOVES & FREQLENCES & IONGUEL:RS & NUM. & MOTS EMPLOYAS & FREQLENCES & LONGLEURS \\
\hline 1 & A & 476 & ! & 12 & AVEC & 19 & 4 \\
\hline 2 & ACTUELLE & 67 & 8 & 13 & BEAUCOUP & 42 & 8 \\
\hline 3 & AMOUR & 21 & 5 & 14 & C & 125 & 1 \\
\hline 4 & ARGENT & 65 & 6 & 15 & $\mathrm{CA}$ & 33 & 2 \\
\hline 5 & ASSEZ & 69 & 5 & 16 & CAUSE & 46 & 5 \\
\hline 6 & $\mathrm{AU}$ & 145 & 2 & 17 & $\mathrm{CE}$ & 29 & 2 \\
\hline 7 & AUCUNE & 15 & 6 & 18 & CELA & 20 & 4 \\
\hline 8 & AUSSI & 17 & 5 & 19 & CHACUN & 40 & 6 \\
\hline 9 & AUTRE & 40 & 5 & 20 & CHANGE & 15 & 6 \\
\hline 10 & $A U X$ & 38 & 3 & 21 & CHANGEMENT & 28 & 10 \\
\hline 11 & AVANT & 76 & 5 & 22 & CHOMAGE & 55 & 7 \\
\hline
\end{tabular}


Análisis estadístico de datos de encuesta

\begin{tabular}{|c|c|c|c|c|c|c|c|}
\hline NLM. & MOTS EMLLOVES & FREQUENCES & WONGUEURS & NLM. & HOTS EMPLLOYES & FREQQUENCES & LONGUEURS \\
\hline 23 & COMME & 35 & 5 & 69 & GRANDE & 36 & 6 \\
\hline 24 & CONCESSIONS & 24 & 1] & 70 & HOMME & 32 & 5 \\
\hline 25 & CONDITIONS & 25 & 10 & 71 & HOMMES & 18 & 6 \\
\hline 26 & CONTRAINTES & 16 & 11 & 72 & IL & 97 & 2 \\
\hline 27 & COUPLE & 74 & 6 & 73 & ILS & 142 & 3 \\
\hline 28 & COUPLES & 50 & 7 & 74 & INCOMPATIBILITE & E 15 & 15 \\
\hline 29 & $\mathrm{D}$ & 269 & I & 75 & INDÉPENDANCE & 107 & 12 \\
\hline 30 & DANS & 101 & 4 & 76 & INDÉPENDANTE & 17 & 12 \\
\hline 31 & $\mathrm{DE}$ & 1180 & 2 & 77 & INDEPENDANTES & 27 & 13 \\
\hline 32 & DES & 407 & 3 & 78 & $\mathrm{~J} E$ & 53 & 2 \\
\hline 33 & DEUX & 37 & 4 & 79 & JEUNE & 36 & 5 \\
\hline 34 & DIFFICILE & 27 & 9 & 80 & JEUNES & 146 & 6 \\
\hline 35 & DIFFICULTÉS & 33 & 11 & 81 & $\mathrm{~L}$ & 322 & 1 \\
\hline 36 & DIVORCE & 63 & 7 & 82 & IA & 760 & 2 \\
\hline 37 & DIVORCER & 45 & 8 & 83 & $I, E$ & 328 & 2 \\
\hline $3 B$ & DONC & 19 & 4 & 84 & LES & 585 & 3 \\
\hline 39 & DU & 143 & 2 & BS & I.EUR & 49 & 4 \\
\hline 40 & EFFORT & 21 & 6 & 86 & LIBERATION & 44 & 10 \\
\hline 41 & EGOISME & 21 & 7 & 87 & I.IBERTE & 166 & 7 \\
\hline 42 & ELLE & 35 & 4 & 88 & IIBRES & 28 & 6 \\
\hline 43 & ELLES & 34 & 5 & 89 & MAINTENANT & 27 & 10 \\
\hline 44 & EMANCIPATION & 23 & 12 & 90 & $\mathrm{MA}$ & 18 & 3 \\
\hline 45 & EN & 137 & 2 & 91 & MAYQUE & 190 & 6 \\
\hline 46 & ENFANTS & 19 & 7 & 92 & MARIAGE & 123 & 7 \\
\hline 47 & ENGAGEMENT & 25 & 10 & 93 & MARIAGES & 27 & 8 \\
\hline 48 & ENSEMBLE & 31 & 8 & 94 & MARIÊ & 28 & 5 \\
\hline 49 & ENTENDENT & 16 & 9 & 95 & MARIENT & 88 & 7 \\
\hline 50 & ENTENTE & 19 & 7 & 96 & MLARIER & 17 & 6 \\
\hline 51 & ENTRE & 34 & 5 & 97 & MAUVAISE & 17 & 8 \\
\hline 52 & EPOUX & 15 & 5 & 98 & MEME & 25 & 4 \\
\hline 53 & ESI & 287 & 3 & 99 & MESENTENTE & 34 & 10 \\
\hline 54 & $\mathrm{ET}$ & 305 & 2 & 100 & MODE & 33 & 4 \\
\hline S5 & ETRE & 43 & 4 & 101 & MODERNE & 32 & 7 \\
\hline 56 & EVOLUTION & 70 & 9 & 102 & MOEURS & 126 & 6 \\
\hline 57 & FACILE & 62 & 6 & 103 & MOINS & 133 & 5 \\
\hline 58 & FACILEMENT & 17 & 10 & 104 & $\mathrm{~N}$ & 128 & l \\
\hline 59 & FACILITÉ & 52 & 8 & 105 & $\mathrm{NE}$ & 282 & 2 \\
\hline 60 & FAIRE & 29 & 5 & 106 & NON & 20 & 3 \\
\hline 61 & FAIT & 68 & 4 & 107 & ON & 177 & 2 \\
\hline 62 & FAMILLE & 23 & 7 & 108 & ONT & 77 & 3 \\
\hline 63 & FEMME & 220 & 5 & 109 & OU & 36 & 2 \\
\hline 64 & FEMMES & 205 & 6 & 110 & PAR & 32 & 3 \\
\hline 65 & FINANCIERE & 39 & 10 & 111 & PARCE & 96 & 5 \\
\hline 66 & FINANCIERS & 17 & 10 & 112 & I'AS & 284 & 3 \\
\hline 67 & FONT & 25 & 4 & 113 & PATIENCE & 24 & 8 \\
\hline 68 & GENS & 228 & 4 & 114 & ['ERSONNE & 15 & 8 \\
\hline
\end{tabular}


"Papers»: Revista de Sociologia

\begin{tabular}{|c|c|c|c|c|c|c|c|}
\hline NUM. & MOTS EMPLOYES & FREQUENCES & LONGUEURS & NUM. & MOTS EMPI,OYES & EREQQUENCES & LONGUEURS \\
\hline 115 & PEUT & 33 & 4 & 138 & SON & 40 & 3 \\
\hline 116 & PLUS & 533 & 4 & 139 & SONT & 122 & 4 \\
\hline 117 & POUR & 94 & 4 & 140 & SOUVENT & 21 & 7 \\
\hline 118 & PROBLEME & 20 & 8 & 141 & SUR & 21 & 3 \\
\hline 119 & PROBLEMES & 34 & 9 & 142 & TEMPS & 31 & 5 \\
\hline 120 & QU & 150 & 2 & 143 & TOT & 21 & 3 \\
\hline 121 & QUAND & 21 & 5 & 144 & Tour & 40 & 4 \\
\hline 122 & QUE & 169 & 3 & 145 & TRAVAIL & 99 & 7 \\
\hline 123 & QUl & 114 & 3 & 146 & TRAVAILLE & 39 & 9 \\
\hline 124 & REFLECCHIR & 19 & 9 & 147 & TRAVAILLENT & 67 & 11 \\
\hline 125 & RESPECT & 17 & 7 & 148 & TROP & 330 & 4 \\
\hline 126 & RESPONSABILITES & 21 & 15 & 149 & UN & 117 & 2 \\
\hline 127 & RIEN & 20 & 4 & 150 & UNE & 86 & 3 \\
\hline 128 & RYTHME & 16 & 6 & $15 !$ & VA & 20 & 2 \\
\hline 129 & S & 97 & 1 & 152 & VALEURS & 20 & 7 \\
\hline 130 & $S A$ & 25 & 2 & 153 & VEULENT & 46 & 7 \\
\hline 131 & SAIS & 22 & 4 & 154 & VEUT & 38 & 4 \\
\hline 132 & SAIT & 31 & 4 & 155 & VIE & 341 & 3 \\
\hline 133 & SANS & 52 & 4 & 156 & VIS & 18 & 3 \\
\hline 134 & $\mathrm{SE}$ & 280 & 2 & 157 & VITE & 43 & 4 \\
\hline 135 & SENS & 17 & 4 & 158 & VIVRE & 47 & 5 \\
\hline 136 & SEXUELLE & 16 & 8 & 159 & $Y$ & 83 & 1 \\
\hline 137 & SOCIETE & 53 & 7 & & & & \\
\hline
\end{tabular}

TABLA 2

Segmentos repetidos del corpus

TABLEAU DES SEGMENTS REPETÉS

SEUUIS MINIMUMM DE TRREQUENCE DE REPETTITION:

SEUIL GENE:RAL

SEGMENTS DE LONGLELR 2

SEGMENTS DE LONGUEUR 3

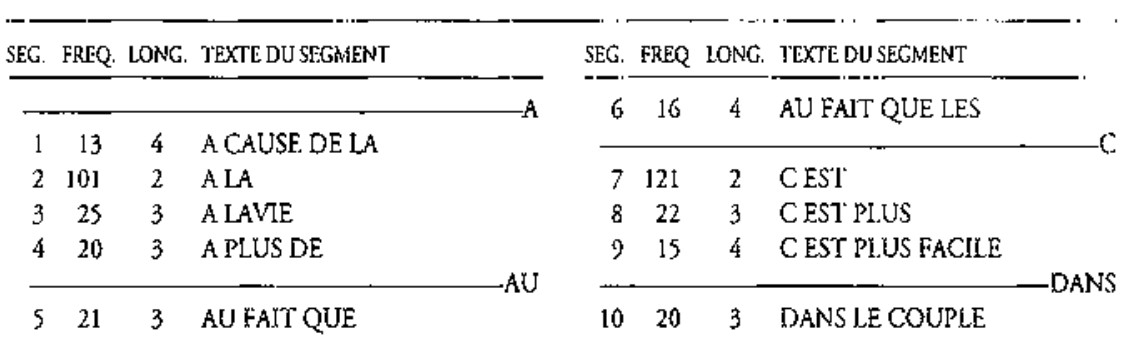


Análisis estadístico de datos de encuesta

SEC. FREQ. LONG. TEXTE DU SEGMENT

\begin{tabular}{rrrl}
\hline 11 & 254 & 2 & DE LA \\
12 & 119 & 3 & DE LA FEMME \\
13 & 58 & 3 & DE LA VIE \\
14 & 12 & 4 & DE LA VIE ACTUELLE \\
15 & 19 & 4 & DE MOINS EN MOINS \\
16 & 48 & 4 & DE PLUS EN PLUS \\
\hline 17 & 13 & 4 & EMANCIPATION DE LA FEMME \\
\hline 18 & 24 & 3 & EST PLUS FACILE \\
19 & 2 & 4 & EST PLUS FACILE DE \\
\hline 20 & 20 & 3 & EVOLLTION DE LA \\
21 & 23 & 3 & FVOLLTION DES MOEURS \\
\hline 22 & 23 & 3 & FAIT QUE LES \\
23 & 10 & 4 & FAT QUE LES FEMMES \\
\hline 24 & 20 & 3 & FEMMES QUI TRAVALLENT \\
\hline 25 & 38 & 3 & GENS SE MARIEN' \\
26 & 21 & 4 & GENS SE MARIEN'I TROP \\
27 & 0 & 5 & GENS SE MARIENT TROP JEUNES \\
\hline 28 & 21 & 3 & IL N Y \\
29 & 20 & 4 & IL N Y A \\
30 & 14 & 5 & IL N Y A PLUS \\
31 & 10 & 6 & IL N Y A PLUS DE \\
32 & 43 & 3 & ILY Y \\
\hline 33 & 22 & 3 & ILS SE MARIENT \\
34 & 15 & 4 & ILS SE MARIENT TROP \\
35 & 12 & 5 & ILS SE MARENT TROP JEUNES \\
\hline 36 & 13 & 4 & INDÉPENDANCE DE LA \\
37 & 11 & 4 & INDEPENDANCE \\
& & & FINANCIERE DES FEMMES \\
\hline
\end{tabular}

\begin{tabular}{rrrl}
\hline 38 & 13 & 4 & JE NE SAIS PAS \\
\hline 39 & 12 & 4 & JEUNES SE MARIENT TROP \\
\hline 40 & 209 & 2 & LA FEMME \\
41 & 20 & 3 & LA FEMME TRAVAILLE
\end{tabular}

SEG. FREQ. LONG. TEXTE DU SEGMENT

\begin{tabular}{|c|c|c|c|}
\hline 42 & 11 & $s$ & $\begin{array}{l}\text { LA LIBERATION DE LA } \\
\text { FEMME }\end{array}$ \\
\hline 43 & 189 & 2 & LA VIE \\
\hline 44 & 34 & 3 & LA VIE ACTUELLE \\
\hline 45 & 21 & 3 & LA VIE MODERNE \\
\hline 46 & $\therefore 2$ & 4 & LE MANQUE D ARGENT \\
\hline 47 & $\therefore 3$ & 4 & LE MARLAGE N EST \\
\hline 48 & $\therefore 2$ & 4 & LE TRAVAIL DES FEMMES \\
\hline 49 & 101 & 2 & LES FEMMES \\
\hline so & 22 & 3 & LES FEMMES SONT \\
\hline \$1 & 10 & 4 & LES FEMMES SONT PIUS \\
\hline 52 & 188 & 2 & LES GENS \\
\hline 53 & 48 & 3 & LES GENS NE \\
\hline 54 & 49 & 3 & LES GENS SE \\
\hline 55 & 37 & 4 & LES GENS SE MARIENT \\
\hline 56 & 20 & 5 & LES GENS SE MARJENT TROP \\
\hline 57 & 24 & 3 & LES GENS SONT \\
\hline 58 & 15 & 4 & LES JEUNES SE MARIENT \\
\hline 59 & 11 & 5 & $\begin{array}{l}\text { LES JEUNES SE MARIENT } \\
\text { TROP }\end{array}$ \\
\hline
\end{tabular}

$60 \quad 224$ LIBÉRATION DE LA FEMME

6116 4 LIBLRTÉ DE LA FEMME

62243 IIBERTÉ DES MOEURS

MANQUE

63283 MANQUE D ARGENT

6412.42 MANQUE DE

\begin{tabular}{cccl}
65 & 10 & 4 & MARLAGE N EST PLUS \\
\hline 66 & 24 & 3 & MODE DE VIE \\
\hline 67 & 23 & 3 & N EST PLUS \\
\hline
\end{tabular}

$\begin{array}{llll}68 & 10 & 4 & \text { NES ENTENDENT PAS }\end{array}$

69303 NESAIT PAS

\begin{tabular}{cccl}
\hline 70 & 26 & 3 & PARCE QUE LES \\
71 & 13 & 4 & PARCE QUE LES GENS \\
\hline 72 & 49 & 3 & PLUS EN PLUS \\
73 & 13 & 4 & PLUS FACILE DE DIVORCER \\
\hline
\end{tabular}


"Papers": Revista de Sociologia

\begin{tabular}{|c|c|c|c|c|c|c|c|}
\hline \multirow{2}{*}{$\frac{\text { SEG. }}{74}$} & \multicolumn{2}{|c|}{ FREQ. LONG } & \multirow{2}{*}{$\begin{array}{l}\text { TEXTE DU SEGMENT } \\
\text { QU IL Y A }\end{array}$} & \multicolumn{4}{|c|}{ SEG. FREQ. LONG. TEXTE DU SEGMENT } \\
\hline & 14 & 4 & & 81 & 14 & 4 & SE MARIENT TROP VTTE \\
\hline 75 & 12 & 4 & QUE LA FEMME TRAVAILLE & 82 & 13 & 4 & TRAVAIL DE LA FEMME \\
\hline 76 & 23 & 3 & QUE LES PEMMES & 83 & 29 & 3 & TRAVAIL DES FEMMES \\
\hline 77 & 24 & 3 & QUE LES GENS & & & & -TROP \\
\hline 78 & 10 & 4 & QUE LES GENS NE & 84 & 26 & 3 & TROP DE IIBERTE \\
\hline 79 & 55 & 3 & SE MARIENT TROP & 85 & 21 & 3 & YAPLUS \\
\hline 80 & 31 & 4 & SE MARIENT TROP JEUNES & 86 & 16 & 4 & YAPLUS DE \\
\hline
\end{tabular}

\section{TABLAS LEXICAS Y SEGMENTALES}

Para aplicar el análisis de correspondencias a las respuestas abiertas, se construyen tablas de contingencia particulares:

1. La tabla léxica contiene la frecuencia con la cual una forma gráfica es empleada por cada uno de los individuos. El análisis de correspondencias, aplicado a esta tabla de frecuencias, llamada tabla léxica, procede por comparación de las distribuciones de las formas en los individuos, es decir compara los perfiles léxicos de los individuos.

2. Si existen una o varias particiones pertinentes del corpus - partición del corpus en grupos de respuestas según la clase de edad del individuo, según el sexo, etc.- se puede construir la tabla de contingencia que contiene la frecuencia de cada forma en cada parte del corpus. Esta tabla se llama tabla léxica agregada.

3. Tablas similares se obtienen sustituyendo las formas por los segmentos repetidos.

\section{ANALISIS DE LA TABLA LÉXICA Y ASOCIACION ENTRE EL VOCABULARIO Y LAS CARACTERISTTCAS DE LOS INDIVIDUOS}

En una tabla de contingencia, las filas y las columnas representan dos particiones de una misma población y ambas particiones juegan un papel análogo: para analizar el contenido de la tabla tiene sentido considerar tanto la nube de puntos-fila como la nube de puntos-columna. El análisis de correspondencias ofrece una representación gráfica conjunta de ambas; para ello efectúa la proyección de las nubes sobre subespacios de dimensión reducida pero manteniendo la máxima dispersión posible. 
El análisis de correspondencias de la tabla Respuestas*Formas proporciona una visualización de la dispersión del vocabulario sobre los primeros ejes. Dos formas próximas habrán sido pronunciadas frecuentemente por los mismos individuos. Las formas alejadas del centro de gravedad, que están en la periferia sobre las gráficas de los planos factoriales, son formas cuyo empleo o cuya frecuencia de empleo diferencian a los individuos. Se puede, así, detectar asociaciones entre formas. En el gráfico 1, se representa el plano factorial principal del análisis de la tabla léxica.

El análisis de dicho gráfico presenta rasgos particulares: las respuestas cortas se distinguen más por la presencia o ausencia de formas gráficas que por Ia diferencia de sus perfiles de frecuencia. Esto hace que las distancias interindividuos sean difíciles de interpretar. Además, la información se reparte sobre numerosos ejes, lo que dificulta su aprehensión global. Se puede decir que, en este primer análisis, se reagrupan e interpretan las respuestas idénticas o similares repetidas con una cierta frecuencia, dejando para otro tipo de análisis las respuestas más originales. Se trata de $\epsilon$ fecruar un trabajo preparatorio, encaminado a establecer un criterio de agrupamiento de las respuestas.

Este tratamiento exploratorio puede ser completado y guiado con ta utilización de dos informaciones suplementarias: las respuestas a las preguntas cerradas y los segmentos repetidos. La primera proporciona una herramienta poderosa para detectar relaciones entre las características de los individuos y su lenguaje; la segunda contextualiza el empleo de las formas y precisa los argumentos empleados por los individuos y como son expresados.

En el ejemplo tratado, se proyectan sobre las gráficas factoriales las modalidades de 8 preguntas cerradas (véase gráfico 3). Dichas modalidades son consideradas columnas suplementarias del análisis anterior. Los indicadores estadísticos, llamados valores-test, calculados por SPAD.T miden, en desviaciones-tipo, cuán lejos del centro de gravedad se situa una modalidad sobre un eje dado: dicho valor-test está normado de tal forma que se puede leer como una realización de una variable norma centrada y reducida, bajo la hipótesis de repartición al azar de las modalidades sobre el eje. Por lo tanto, se considera relacionada con el eje una modalidad cuyo valor-test asociado es mayor que 1.96 o menor que -1.96. En efecto, bajo la hipótesis de repartición aleatoria de las modalidades, la probabilidad de que el valor-test esté entre estos dos valores es del $95 \%$ (véase tabla 3).

Los gráficos 2 y 3 muestran el posicionamiento de las características de los individuos y de los segmentos repetidos sobre el plano factorial del gráfico 1. Formas, segmentos y características individuales son puntos de un mismo espacio, lo que legitima interpretar la proxirnidad entre dos puntos. La lectura simultánea de las tres figuras permite ver las características de los individuos que emplean un cierto argumento, con qué palabras y con qué cons- 
Coordenadas y Valores-test de modalidades sobre los ejes factoriales

COORDOONNEESS ET VALEURS-TEST DES MODALITESS SUR LES AXES 1 A 3

NOTE : LES DISTANCES A LORIGINE SONT DIVTSEES PAR 10.

\begin{tabular}{|c|c|c|c|c|c|c|c|c|c|c|c|c|c|}
\hline \multicolumn{4}{|c|}{ MODALITES } & \multicolumn{5}{|c|}{ COORDONNÉES } & \multicolumn{5}{|c|}{ VALEURS-TEST } \\
\hline IDEN - LIBELIE & EFF. & P.ABS & DISTO & 1 & 2 & 3 & 0 & 0 & 1 & 2 & 3 & 0 & 0 \\
\hline \multicolumn{14}{|l|}{ 4. Sexe de lenqueté } \\
\hline AD01 - masculin & 933 & 6344.00 & 0.13 & -0.03 & 0.04 & 0.01 & 0.00 & 0.00 & -3.6 & 4.8 & 1.5 & 0.0 & 0.0 \\
\hline $\mathrm{AD} 02$ - feminin & 1067 & 8022.00 & 0.08 & 0.03 & -0.04 & -0.01 & 0.00 & 0.00 & 3.6 & .4 .8 & -1.5 & 0.0 & 0.0 \\
\hline \multicolumn{14}{|c|}{ 10. A2a: avez-yous eat des enfants? } \\
\hline Al01 - oui & 1378 & 9881.00 & 0.05 & -0.01 & -0.01 & .0 .01 & 0.00 & 0.00 & -1.3 & -1.3 & -1.6 & 0.0 & 0.0 \\
\hline AI02 - non & 622 & 4485.00 & 0.22 & 0.02 & 0.02 & 0.02 & 0.00 & 0.00 & 1.3 & 1.3 & 1.6 & 0.0 & 0.0 \\
\hline \multicolumn{14}{|c|}{ 1S. CI: la famille est le seul endroit où lon se sent bien } \\
\hline Al01 - fam. endroit bien & 1225 & 8617.00 & 0.07 & 0.08 & -0.02 & 0.02 & 0.00 & 0.00 & 11.3 & -2.9 & 3.5 & 0.0 & 0.0 \\
\hline AL02 - Gamm. non endroir bien & 774 & 5748.00 & 0.15 & -0.12 & 0.03 & -0.04 & 0.00 & 0.00 & -11.3 & 3.0 & -3.5 & 0.0 & 0.0 \\
\hline AL03- famille n.s.p. & 1 & 1.00 & 1436.50 & -1.10 & -0.42 & 0.50 & 0.00 & 0.00 & -1.1 & -0.4 & -0.5 & 0.0 & 0.0 \\
\hline \multicolumn{14}{|l|}{ 16. C2: opinion sur le mariage } \\
\hline AM01 - indissoluble & 440 & 3048.00 & 0.37 & 0.10 & -0.03 & 0.03 & 0.00 & 0.00 & 6.4 & -1.8 & 2.1 & 0.0 & 0.0 \\
\hline AM02 - dissout si pb. grave & 714 & 5311.00 & 0.17 & 0.03 & 0.01 & -0.06 & 0.00 & 0.00 & 2.5 & 1.1 & -5.1 & 0.0 & 0.0 \\
\hline AM03 - dissout si accord & 764 & 5499.00 & 0.16 & -0.10 & 0.02 & 0.04 & 0.00 & 0.00 & -9.7 & 1.9 & 3.4 & 0.0 & 0.0 \\
\hline AM04 - mariage n.s.p. & 82 & 508.00 & 2.73 & 0.21 & -0.18 & -0.01 & 0.00 & 0.00 & 4.8 & -4.1 & -0.2 & 0.0 & 0.0 \\
\hline
\end{tabular}


Análisis estadístico de datos de encuesta

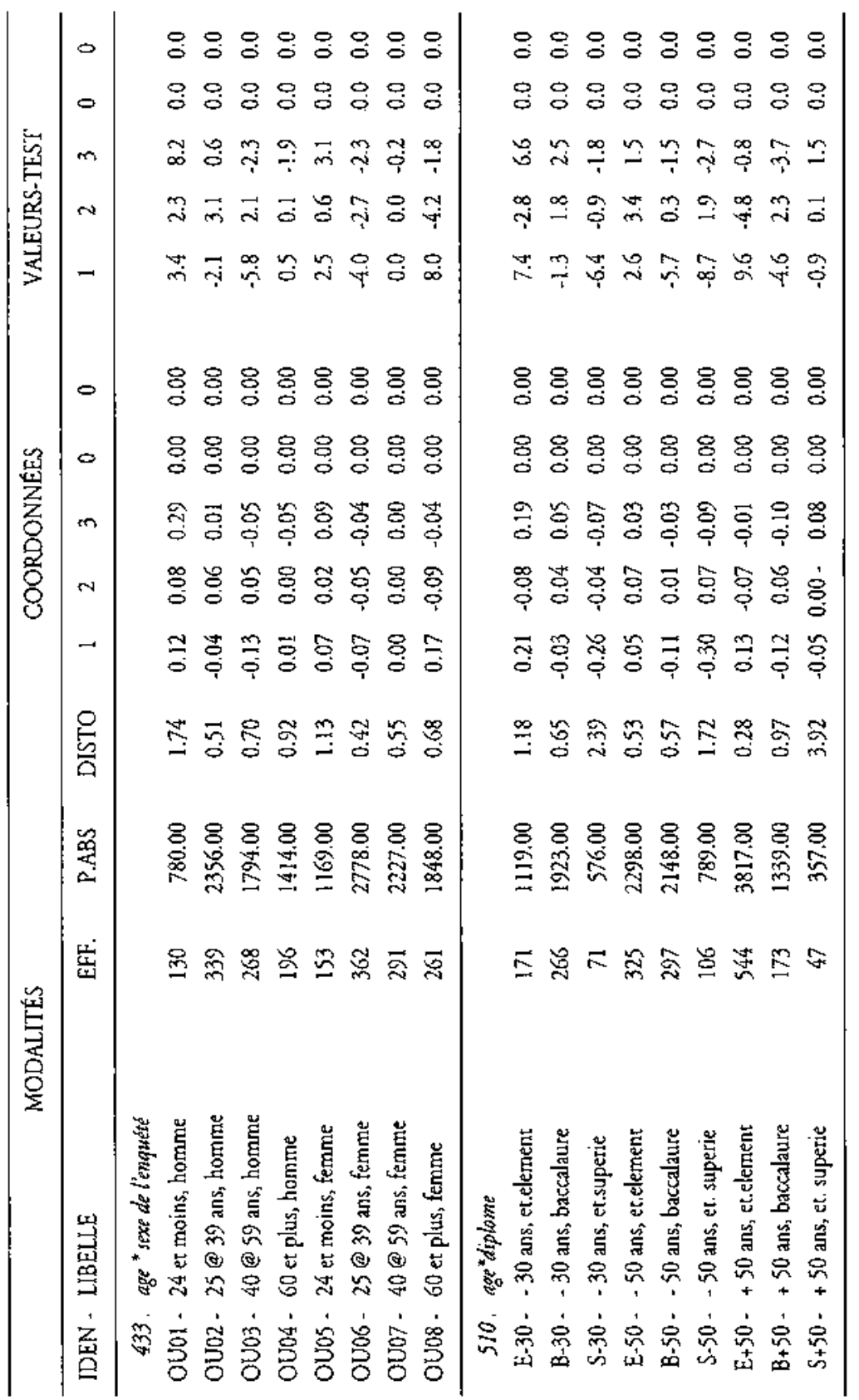




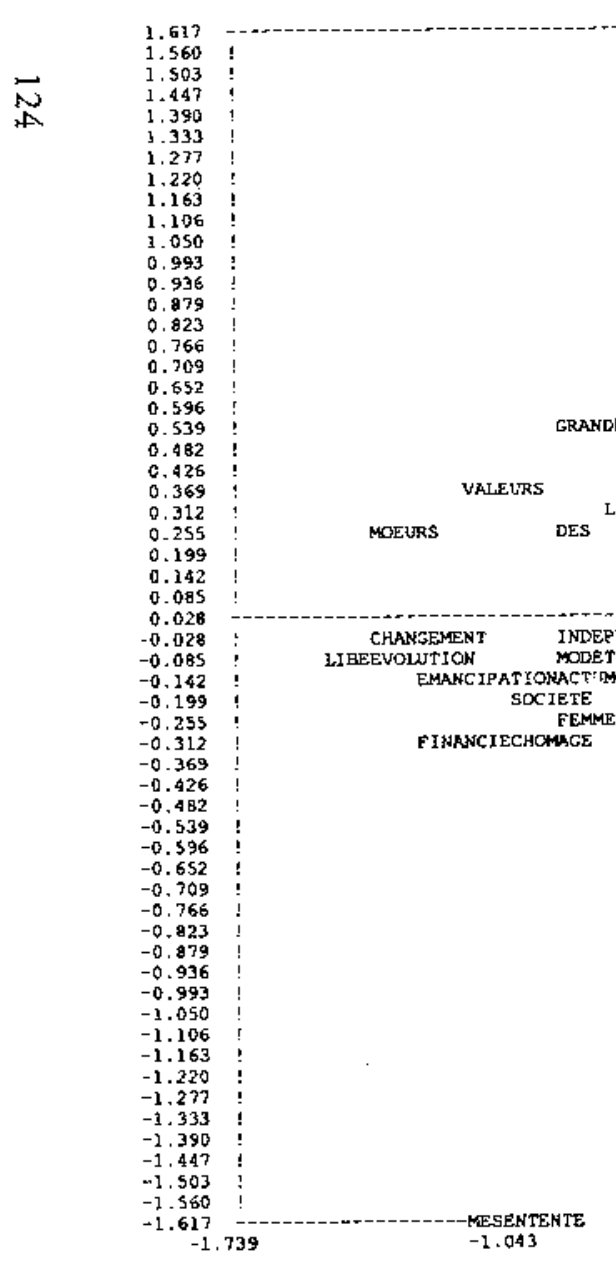

-MARTAGES

----JEUNE

TOT

.255

LIBERTE RUX

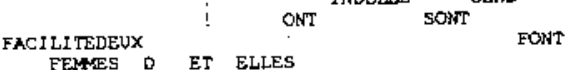

ARGEAMOUPROBDIVOAAINCOUPLES ETREENGAAVANT

Fon

Ils DIFEICULPAR AUTRELUSVIVRE OU PARCE ENSEMBIE NDEPENDANCE A AU DIVOEN TEMPFAITPOUR

DANS CELA NA DUAND ASSE?

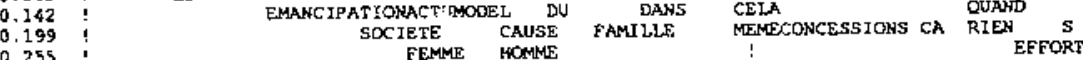

FEMME HOMME HOMME

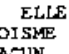

TOUTPEUT VA FAIRE

$$
\text { CHATUN }
$$

COUEIE

$\begin{array}{lll}\text { VEUT } & \vdots & \text { NON }\end{array}$

MARIENT

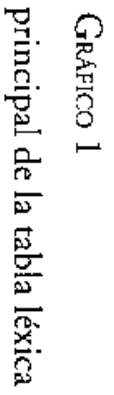

JE.

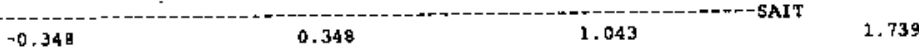




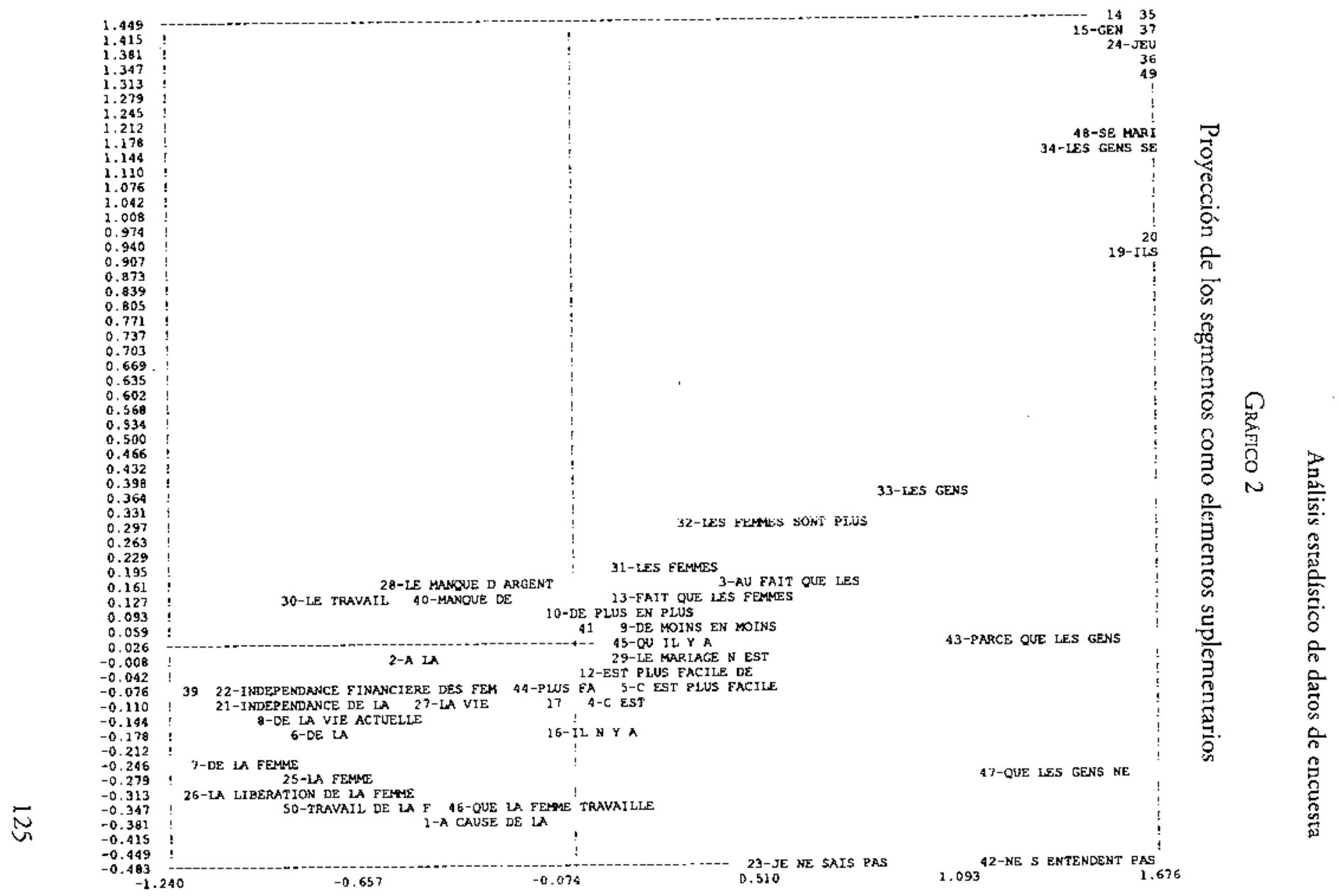


"Papers»: Revista de Sociologia

\section{GRÄFICO 3}

Modalidades proyectadas sobre el plano factorial Forma*Individuos

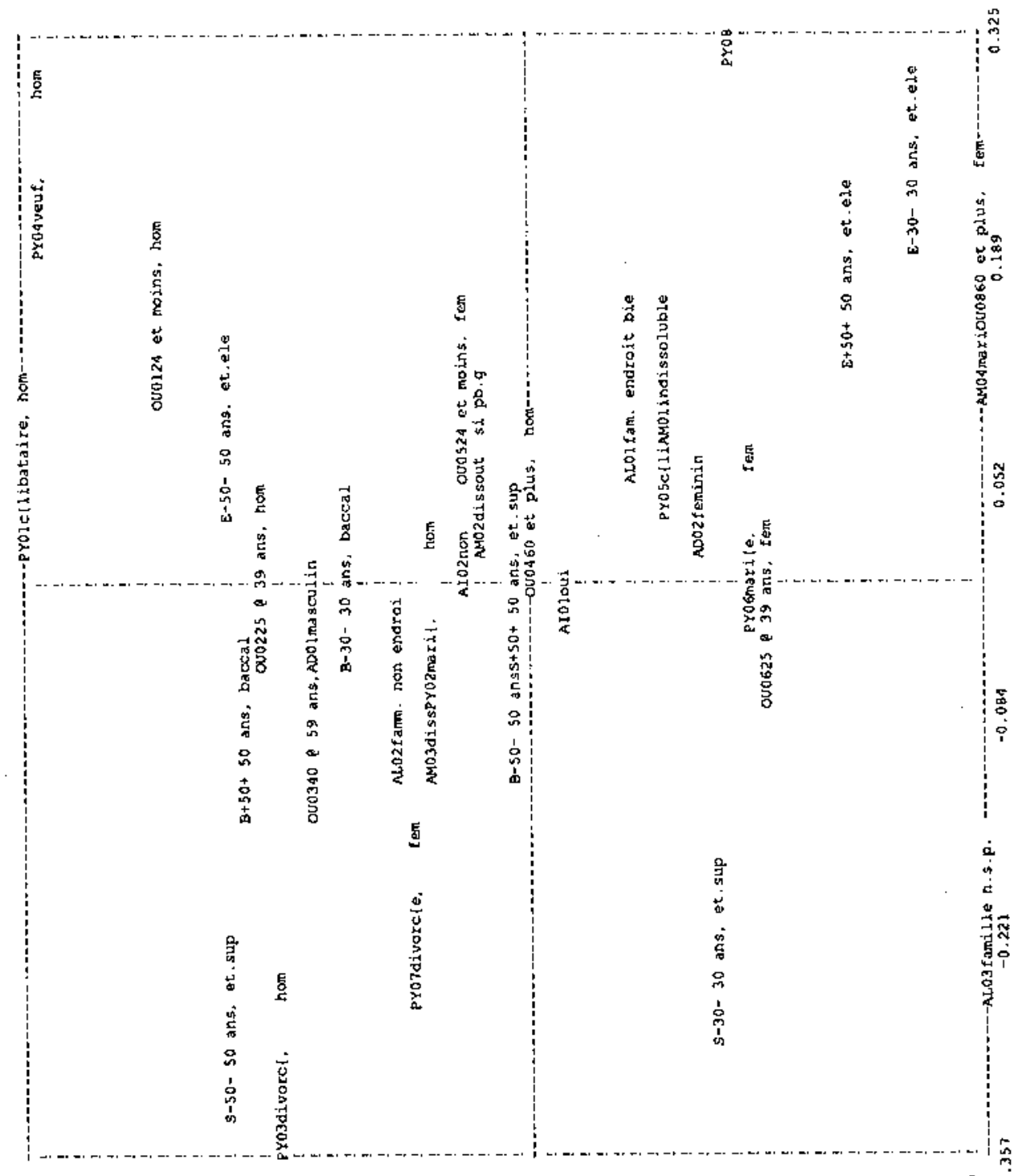

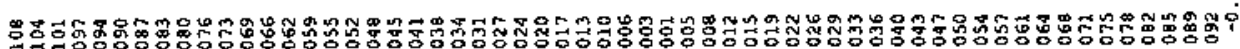

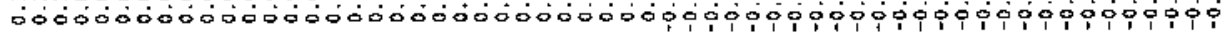




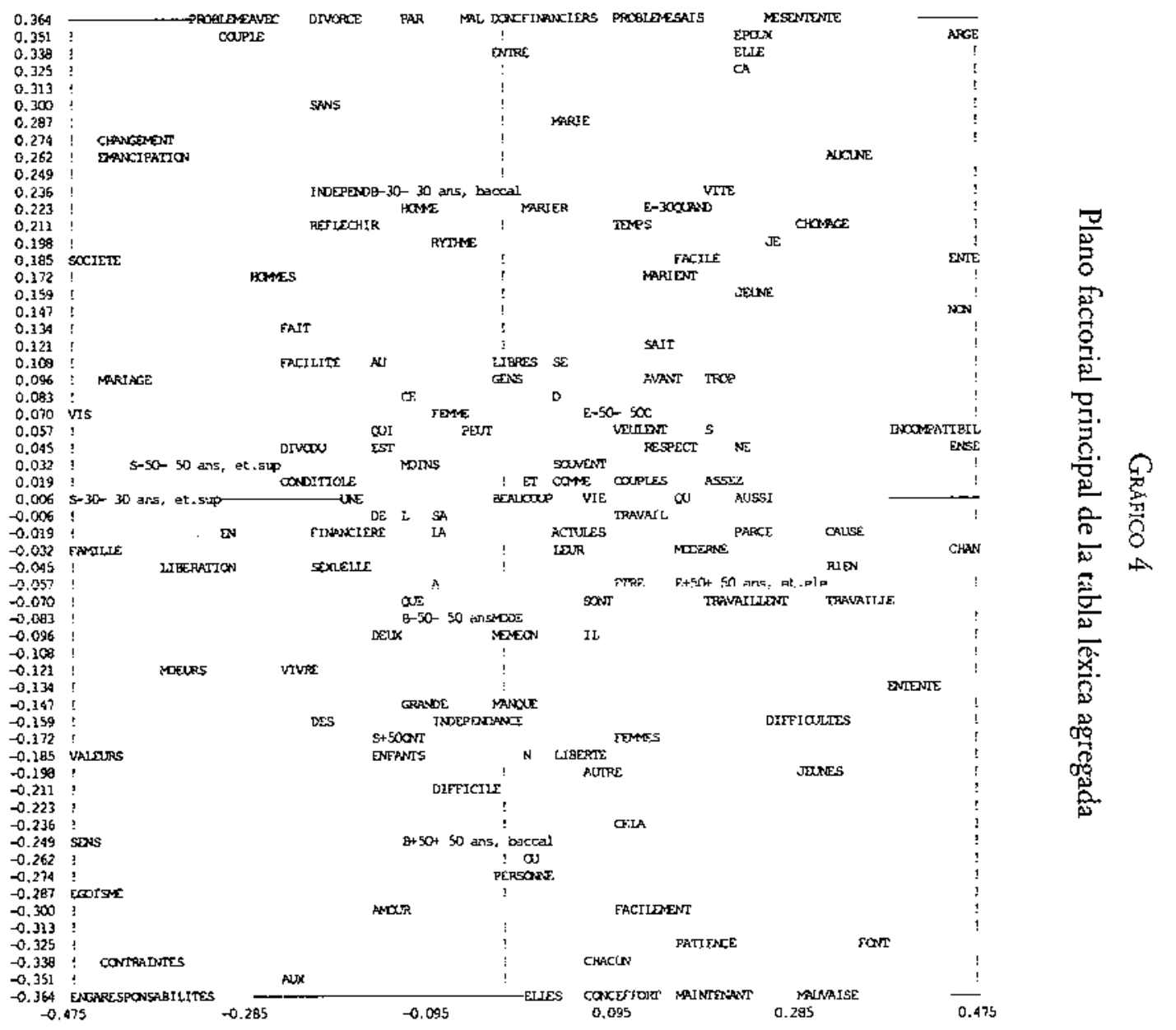


"Papers": Revista de Sociologia

trución sintáctica lo expresan, es decir permite ver "quién dice qué y como lo dice». Por ejemplo, se pone de manifiesto la asociación entre un nivel de estudios bajo y la no respuesta dada por Je ne sais pas, entre un nivel de estudios alto y las respuestas que hablan de la liberación de la mujer, del cambio de las costumbres.

\section{ANALISIS DE UNA TABLA LEXICA AGREGADA Y FORMAS CARACTERISTICAS}

El tratamiento estadístico de grupos de respuestas tiene mucho más interés que el de las respuestas individuales. Los análisis presentados en los párrafos anteriores constituyen una ayuda para la selección de un cricerio de agrupamiento pertinente.

Entre todas las variables cualitativas consideradas, se puede escoger una de ellas y construir la tabla léxica agregada correspondiente. Escoger una variable supone escoger un punto de vista; las estructuras que se observarán habrán sido, en parte, suscitadas por esta elección: la dispersión del vocabulario en función de la pertenencia a una u otra categoría de esta variable constituirá la trama de fondo sobre la cual se superpondrán otras estructuras. Será, en cierto sentido, una trama de referencia.

Un criterio pertinente para el agrupamiento de las respuestas individuales es la modalidad de la variable Título*Edad. La tabla léxica agregada obtenida contiene las frecuencias con la cual cada una de las formas ha sido empleada por cada una de las 9 categorías de individuos.

El análisis de esta tabla consiste en comparar los perfiles lexicales de las distintas categorias de individuos. Se proponen tres herramientas para efectuar este análisis: el análisis de correspondencias, las listas de formas características y las listas de respuestas características.

\section{ANALISIS DE CORRESPONDENCIAS DE LA TABLA LEXICA AGREGADA}

En el gráfico 4, se presenta el plano factorial principal del análisis de la tabla léxica agregada. Para facilitat la lectura de la gráfica, se puede unir con líneas continuas las modalidades que indican una edad parecida, y por líneas discontinuas las modalidades que indican un mismo nivel de título

Se puede interpretar el primer eje como un eje de titulación: la progresión del nivel de la titulación de izquierda a derecha del eje define una trayectoria extraordinariamente próxima al eje en casi toda su extensión. El segundo eje 
parece ser un eje de edad. Opone los más jovenes - arriba-a a los mayores. Más que proyectar como elemento suplementario la variable "Edad", es preferible proyectar la variable "Edad*Sexo". La notable similaridad de las dos trayectorias de edad en el caso de los jovenes tiende a desaparecer en el caso de los mayores. Existen de hecho correlaciones entre las variables utilizadas, en particular entre el sexo, la edad y el título: la mujer mayor suele tener menor titulación que el hombre mayor. Por es:o el punto-modalidad «Mujer mayor" está atraido por el nivel de estudios bajo. Recordemos que el punto de la gráfica asociado a una modalidad se encuentra en el centro de gravedad de las formas utilizadas por el grupo de individuos que poseen esta modalidad.

\section{SELECCION DE FORMAS CARACTERISTICAS}

Se puede completar la representación gráfica obtenida por la selección de las formas más características de cada uno de los 9 grupos determinados por la variable Edad-Título. Esta selección, apoyada sobre criterios probabilistas, derecta las formas "anormalmente" frecuentes en las respuestas de un grupo de individuos. Para facilitar la lectura de la caracterización de un grupo por una forma, el sistema SPAD.T asocia a cada forma un valor-test que mide la diferencia entre la frecuencia de la forma en el grupo y la frecuencia de la misma forma en la población.

De la misma forma que antes, dicho valor-test está normado de tal forma que se puede leer como una realización de una variable norma centrada y reducida, bajo la hipótesis de repartición aleatoria de la forma considerada en las clases. Por lo tanto, se consideran caracterísricas de una clase las formas cuyo valor-test asociado es mayor que 1.96 (formas sobrerepresentadas en la clase) o menor que -1.96 (formas subrepresentadas en la clase). En la Tabla 4, se muestran las formas características de 4 de los 9 textos formados según las 9 modalidades de la variable Título*Edad.

\section{SELECCION DE RESPUESTAS CARACTERIISTICAS}

Dado un grupo de individuos, se puede calcular el perfil léxico medio del grupo, a partir de los perfiles léxicos de los individuos que lo componen. Se puede considerar como características de un grupo, las respuestas más próximas a este perfil medio, próximas en el sentido de la distancia de Chi-2, distancia entre distribuciones de frecuencias ya utilizada en el análisis de correspondencias. 
"Papers»: Revista de Sociologia

$$
T_{A B L A} 4
$$

Formas características de 4 textos:

Menores de 30 años sin estudios y con estudios superiores.

SELLECTION DES FORMES LEXICALES CARACTERISTIQUES

TEXTE NUMERO $1 \mathrm{E}-30=-30$ ans, et.element

\begin{tabular}{llccrccc}
\hline & $\begin{array}{l}\text { Libelle de la } \\
\text { forme graphique }\end{array}$ & \multicolumn{2}{c}{ Pourcentage } & \multicolumn{2}{c}{ Fréquence } \\
Interne & Global & Interne & Globale & V. test & Proba \\
\hline 1 & SAIS & 0.78 & 0.15 & 9. & 22. & 4.097 & 0.000 \\
2 & ARGENT & 1.29 & 0.44 & 15. & 65. & 3.676 & 0.000 \\
3 & PROBLEMES & 0.69 & 0.23 & 8. & 34. & 2.650 & 0.004 \\
4 & COUPLE & 1.12 & 0.50 & 13. & 74. & 2.602 & 0.005 \\
5 & JE & 0.86 & 0.36 & 10. & 53. & 2.436 & 0.007 \\
& & & & & & & \\
5 & ON & 0.69 & 1.20 & 8. & 177. & -1.579 & 0.057 \\
4 & AU & 0.52 & 0.98 & 6. & 145. & -1.586 & 0.056 \\
3 & IL & 0.26 & 0.66 & 3. & 97. & -1.666 & 0.048 \\
2 & DIVORCE & 0.00 & 0.43 & 0. & 63. & -2.538 & 0.006 \\
1 & ONT & 0.00 & 0.52 & 0. & 77. & -2.918 & 0.002 \\
\hline
\end{tabular}

TEXTE NUMERO $3 S-30=-30$ ans, et.superie

\begin{tabular}{llcccccc}
\hline & $\begin{array}{l}\text { Libelle de la } \\
\text { forme graphique }\end{array}$ & \multicolumn{2}{c}{ Pourcentage } & \multicolumn{4}{c}{ Frequence } \\
Interne & Global & Interne & Globale & V. test & Proba \\
\hline 1 & DEUX & 1.02 & 0.25 & 6. & 37. & 2.734 & 0.003 \\
2 & MARIAGE & 2.04 & 0.83 & 12. & 123. & 2.686 & 0.004 \\
3 & EN & 2.04 & 0.93 & 12. & 137. & 2.388 & 0.008 \\
4 & RESPONSABILITES & 0.68 & 0.14 & 4. & 21. & 2.381 & 0.009 \\
5 & EST & 3.23 & 1.94 & 19. & 287. & 2.036 & 0.021 \\
& & & & & & & \\
5 & TROP & 1.02 & 2.23 & 6. & 330. & 2.032 & 0.021 \\
4 & PARCE & 0.00 & 0.65 & 0. & 96. & -2.063 & 0.020 \\
3 & JEUNES & 0.17 & 0.99 & 1. & 146. & -2.089 & 0.018 \\
2 & FEMMES & 0.34 & 1.39 & 2. & 205. & 2.302 & 0.011 \\
1 & ILS & 0.00 & 0.96 & 0. & 142. & 2.752 & 0.003 \\
\hline
\end{tabular}


Formas características de 4 textos:

Menores de 50 años sin estudios y con estudios superiores.

SELLECTION DES FORMES LEXICAIES CARACTERISTIQUES

TEXTE NUMERO $1 \mathrm{E}-30=-30$ ans, et.element

\begin{tabular}{llcccccc}
\hline & \multicolumn{2}{l}{$\begin{array}{l}\text { Libelle de la } \\
\text { forme graphique }\end{array}$} & \multicolumn{2}{c}{ Pourcentage } & \multicolumn{3}{c}{ Friquence } \\
Interne & Global & Interne & Globale & V. test & Proba \\
\hline 1 & ILS & 1.56 & 0.96 & 61. & 142. & 4.186 & 0.000 \\
2 & PAS & 2.50 & 1.92 & 98. & 284. & 2.966 & 0.002 \\
3 & NE & 2.48 & 1.91 & 97. & 282. & 2.914 & 0.002 \\
4 & POUR & 0.97 & 0.64 & 38. & 94. & 2.854 & 0.002 \\
5 & CHANGE & 0.23 & 0.10 & 9. & 15. & 2.495 & 0.006 \\
& & & & & & \\
5 & EVOLUTION & 0.23 & 0.47 & 9. & 70. & -2.597 & 0.005 \\
4 & COUPLE & 0.23 & 0.50 & 9. & 74. & -2.840 & 0.002 \\
3 & DE & 6.90 & 7.98 & 270. & 1180. & -2.904 & 0.002 \\
2 & SOCIETE & 0.10 & 0.36 & 4. & 53. & -3.284 & 0.001 \\
1 & MARIAGE & 0.41 & 0.83 & 16. & 123. & -3.509 & 0.000 \\
\hline
\end{tabular}

TEXTE NUMERO $9 \mathrm{~S}+50=+50$ ans, et.superie

\begin{tabular}{|c|c|c|c|c|c|c|c|}
\hline & \multirow{2}{*}{$\begin{array}{l}\text { Libelle de la } \\
\text { forme graphique }\end{array}$} & \multicolumn{2}{|c|}{ Pourcentage } & \multicolumn{2}{|c|}{ Fréquence } & \multirow[b]{2}{*}{ Y. test } & \multirow[b]{2}{*}{ Proba } \\
\hline & & Interne & Global & Interne & Globale & & \\
\hline 1 & $\mathrm{~L}$ & 4.29 & 2.18 & 16. & 322. & 2.417 & 0.008 \\
\hline 2 & RESPECT & 0.80 & 0.11 & 3 & 17. & 2.395 & 0.008 \\
\hline 3 & MARIER & 0.80 & 0.11 & 3. & 17. & 2.395 & 0.008 \\
\hline 4 & QUE & 2.68 & 1.14 & 10. & 169. & 2.302 & 0.011 \\
\hline 5 & RIEN & 0.80 & 0.14 & 3. & 20. & 2.221 & 0.013 \\
\hline 5 & COUPLE & 0.00 & 0.50 & 0. & 74. & -1.046 & 0.148 \\
\hline 4 & LA & 3.75 & 5.14 & 14. & 760 & -1.119 & 0.131 \\
\hline 3 & ILS & 0.27 & 0.96 & 1. & 142. & -1.162 & 0.123 \\
\hline 2 & FEMME & 0.54 & 1.49 & 2. & 220 & -1.398 & 0.081 \\
\hline 1 & TROP & 1.07 & 2.23 & 4. & 330 & -1.421 & 0.078 \\
\hline
\end{tabular}


"Papers": Revista de Sociologia

\section{TABLA 5}

Respuestas características de 4 textos:

Selección según el criterio de frecuencia de las formas

TEXTE NUMERO 1 E-30 $=-30$ ans, et.element

\begin{tabular}{|c|c|}
\hline $\begin{array}{l}\text { Critère de } \\
\text { classification }\end{array}$ & Réponse ou individu caractéristiqque \\
\hline $2.626-1$ & NE SAIS PAS \\
\hline $2.626-2$ & NE SAIS PAS \\
\hline $2.579-3$ & JE NE SAIS PAS \\
\hline $2.579-4$ & EE NE SAIS PAS \\
\hline $2.488-5$ & JE SAIS PAS, NON JE SAIS PAS \\
\hline
\end{tabular}

TEXTE NUMERO 3 S-30 $=-30$ ans, et.superie

\begin{tabular}{|c|c|c|}
\hline $\begin{array}{l}\text { Critere de } \\
\text { classiffcatio }\end{array}$ & & Réponse ou individu caractéristique \\
\hline $1.243-$ & 1 & L'EVOLUTION DES MOEURS \\
\hline $1.237-$ & 2 & $\begin{array}{l}\text { MOINS DE RESPONSABILITES DES DEUX PARTIES VIS A VIS DU } \\
\text { MARLAGE, LIBERATION DES MOEURS, MORALE MOINS STRICTE, }\end{array}$ \\
\hline $1.108-$ & 3 & $\begin{array}{l}\text { MANQUE DE MATURITE AU MOMENT DU MARIAGE, EVOLUTION } \\
\text { DIFFEREN'TE DES DEUX PARTENAIRES }\end{array}$ \\
\hline $1.020-$ & 4 & EVOLUTION DES MOEURS LA FEMME AU TRAVAIL \\
\hline $1.010-$ & 5 & A IA DEGRADATION DES VALEURS MORALES \\
\hline
\end{tabular}

TEXTE NUMERO $7 E+50=+50$ ans, et.element

\begin{tabular}{ll}
\hline $\begin{array}{l}\text { Criterc de } \\
\text { classification }\end{array}$ & Réponse ou individu caractéristique \\
\hline $2.369-1$ & ILS NE S'ENTENDENT PAS \\
$1.960-2$ & NE SAIT PAS \\
$1.960-3$ & NE SAIT PAS \\
$1.960-4$ & NE SAIT PAS \\
$1.960-5$ & NE SAIT PAS \\
\hline
\end{tabular}

TEXTE NUMERO I $S+50=+50$ ans, et.superie

\begin{tabular}{|c|c|}
\hline $\begin{array}{l}\text { Critére de } \\
\text { classification }\end{array}$ & Réponse ou individu caracréristique \\
\hline $1.024-1$ & LIBERALISATION DES MOEURS \\
\hline $1.024-2$ & LIBERALISATION DES MOEURS \\
\hline $1.013-3$ & AU NON RESPECT DES ENGAGEMENTS \\
\hline $0.845-4$ & $\begin{array}{l}\text { L'ENGAGEMENT EST' SUBJECTIF ET LIE A L'ATTRAIT DE L'UN POUR } \\
\text { L'AUTRE ET NON PLUS A L'AMOUR QUE L'UN DOIT AVOIR POUR L'AUTRE }\end{array}$ \\
\hline $0.806 \ldots 5$ & L'AFHAIBLISSEMENT DE L'INSTIYUTION FAMILIAEE \\
\hline
\end{tabular}


Se pueden, también, seleccionar las respuestas características siguiendo otro criterio, el criterio del valor-test medio. Como lo hemos visto en el párrafo anterior, se afecta a cada forma y para cada grupo un valor-test que califica la significación de su frecuencia en el grupo comparada a su frecuencia en la población. Se puede atribuir a cada respuesta la media de los valorestest de las formas que la componen. Las respuestas con valor medio más al to serán las más características del grupo (ver table 5).

\section{CONCLUSION}

Los tratamientos posibles son más numerosos que los aquí propuestos, pero se ha querido explicitar sobre todo la especificidad de los métodos empleados: la aproximación estadística a los datos textuales presentada en este artículo ofrece una nueva lectura de los textos, lectura esencialmente distinta pero complementaria de la lectura humana. Dicha lectura proporciona una descripción cuantitariva, sistemática y exhaustiva del vocabulario. Ofrece una aproximación comparativa: se describen, analizan e interpretan las diferencias entre los textos.

Los datos de encuesta constituyen el terreno de elección de estos métodos. Pero se puede analizar con provecho otro tipo de textos - textos literarios, discursos políticos, entrevistas no directivas, etc. El corpus constituido debe presentar un cierto grado de homogeneidad y de exhaustividad. Los resultados obtenidos facilitan entonces la construcción de hipótesis y orientan los análisis posteriores.

La integración del conjunto de los métodos en un mismo entorno informático, disponible tanto para microcomputadores como para grandes computadores, permite su utilización por todo tipo de usuarios. El coste de la grabación de los datos textuales sobre soporte magnético queda compensado por la calidad del instrumento de observación ofrecido. La codificación minima requerida facilita el tratamiento de textos grabados prealablemente para otros fines.

\section{BIBLIOGRAFIA}

Bécue M. Un sistema informático para el análisis estadistico de datos textuales. Tesis doctoral. Facultat d'Informàtica de Barcelona. UPC. 1989

Benzécri J.P. La taxinomie, vol. I, L'Analyse de Correspondances, vol. II, Dunod. París, 1973. 
"Papers": Revista de Sociologia

Benzécri J.P. «Pratique de l'Analyse des Données", tomo 3, Linguistique \&́ Lexicologie. Dunod. Rarís, 1973.

Bouroche J.M., Saporta G. L'Analyse des Données, "Que sais-je", n०1.854, P.U.F. Paris, 1980.

Brian E. Analyse des Données Lexicométriques. Rapport Credoc/D.G.T., 1984.

Escouffier Y. "L'Analyse des Correspondances, ses Propriétés.ses Extensions". Bull. of the Inst. Stat. Inst., 4, 28-2. 1985 .

Haeusler L. Analyse Lexicale de Réponses Libres: Le Cồt de l'Electricité. Rapport Crédoc-EDF, 1984.

Lafon P., Salem A. "L'Inventaire des Segments Répérés d'un Texte», en Mots $n^{\circ} 6$, pp. 161-177, 1983.

Lebart L., Houzel van Effenterre Y. "Le Système d'Enquêtes sur les Aspirations des Français, Une Brève Présentationm, en Consommation no1, po. 3-25. Dunod, París, 1980.

Lebart L. “L'Analyse Statistique des Réponses Libres dans les Enquêtes Socio-économiques". Consommation, $n^{\circ} 1$, pp. 39-62, Dunod. París, 1982.

Lebart L., Morineau A., Fénelon J.P. Traitement des Données Statistiques. Dunod. París, 1979.

Lebart L., Morineau A., Warwick Multivariate Descriptive Statistical Analysis. I. Wiley and Sons. Nueva York, 1984.

Lebart L., Salem A. Analyse Statistique des Données Textuelles. Dunod. París, 1988.

Lebart L., Morineau A., Bécue M., (con la col. de P. Pleuvret et L. Haeusler) SPAD.T, Systeme Portable pour l'Analyse des Donnés Textuelles. Manuel de Référence. CISIA. Paris, 1989.

Morineau A. "Computational and Statistical Methods of Exploratory Analysis of Textual Data». COMPSTAT, Physica Verlag, Viena, pp 372-377, 1984.

Reinert M. "Un logiciel d'Analyse Lexicale». en Les Cahiers de l'Analyse des Données, 4, pp 471-484. Dunod. París, 1986.

Salem A. "Analyse Factorielle et Lexicométrie. Synthèse de Queląues expériences", Mots na4, pp I47-168. 1982.

Salem A. Pratique des Segments Répétés, Essai de Statistique Textuelle. Klincksieck. París, 1987.

Volle M. Analyse des Données. Economica. París, 1980. 